\title{
Analysis of $M^{[X]} / G / 1$ Queue with Service Interruption and Extended Server Vacations with Bernoulli Schedule
}

\author{
G. Ayyappan \\ Department of Mathematics \\ Pondicherry Engineering College \\ Puducherry, India
}

\author{
K. Sathiya \\ Department of Mathematics \\ Krishna Engineering College \\ Puducherry, India
}

\begin{abstract}
We study a batch arrival queueing system with service interruption and Extended server vacation based on Bernoulli schedule. A single server provides essential service to all arriving customers with service time following general distribution. After every service completion the server has the option to leave for phase one vacation of random length with probability $\mathrm{p}$ or to continue staying in the system with probability 1-p. The new assumption in this paper is that the server go on extended vacation, as soon as the completion of phase one vacation, the server undergoes phase two and phase three vacation. On completion of three heterogeneous phase of vacation the server return back to the system. The vacation times are assumed to be general. The server is interrupted at random and the duration of attending interruption follows exponential distribution. Also we assume, the customer whose service is interrupted goes back to the head of the queue where the arrivals are Poisson. Using supplementary variable technique, the Laplace transforms of time dependent probabilities of system state are derived. From this we deduce the steady state results. We also obtain the average queue size and average waiting time.
\end{abstract}

\section{General Terms:}

Subject Classification (AMS) : 60K25, 60K30

\section{Keywords:}

Batch arrival, Transient state solution, Extended vacation time, Average queue size, Average waiting time

\section{INTRODUCTION}

Vacation queues have been investigated for over two decades as a very useful tool for modeling and analyzing computer systems, communication networks, manufacturing and production systems and many others.

Queuing systems with server vacations and/or random system breakdowns have been studied by numerous researchers including the survey of Doshi [5], Kulkarni and Choi [6], Takagi [9], Takine and Sengupta [10], Wang et al. [15], Madan et al. [7], Tian and Zhang [13], Maraghi et al. [8] and Thangaraj and Vanitha [11]. However, in these models, the server stops the original work in the vacation period and can not come back to the regular busy period until the vacation period ends.

In queueing theory periods of temporary service unavailability are referred to as server vacations, server interruptions or server breakdowns. Queueing models with service interruptions have proved to be a useful abstraction in situations where a service facility is shared by multiple queues or where the facility is subject to failure.

White and Christie [16] have studied queues with service interruptions. They consider an M/M/1 queueing system with exponentially distributed interruptions. Generally distributed service times and interruptions are considered by Avi-Itzhak and Naor [1], Thiruvengadam [12], Baskar et al. [2], Balamani [3]. Vacation queues with c servers have been studied by Tian et al. [14]. Borthakur and Choudhury [4] have studied vacation queues with batch arrivals. We assume that the customers arrive to the service station in batches of variable size, but are served one by one. We assume that the service times, vacation times, have a general distribution while the time to interruptions is exponentially distributed.

Most of the recent studies have been devoted to batch arrival vacation models under different vacation policies because of its interdisciplinary character. In this paper, we consider a batch arrival queueing system $M^{[X]} / G / 1$ with service interruption, in which we assume that after every service completion, the server has the option to leave for a vacation of random length with probability p or to continue staying in the system with probability 1-p. The vacation period has three heterogeneous phases. On completion of three vacation phases the server return back to the system. Also we assume, the customer whose service is interrupted goes back to the head of the queue where the arrivals are Poisson.

This paper is organized as follows. The mathematical description of our model is given in section 2. Definitions and Equations governing the system are given in section 3 . The time dependent solution have been obtained in section 4 and corresponding steady state results have been derived explicitly in section 5. Average queue size and average waiting time are computed in section 6 and 7 respectively. Particular case is discussed in section 8. Conclusion are given in section 9 .

\section{MATHEMATICAL DESCRIPTION OF THE MODEL}

We assume the following to describe the queueing model of our study.

a) Customers arrive at the system in batches of variable size in a compound Poisson process and they are provided one by one service on a first come - first served basis. Let $\lambda c_{i} d t(i=1,2, \ldots$ .) be the first order probability that a batch of $i$ customers arrives at the system during a short interval of time $(t, t+d t]$, where $0 \leq c_{i} \leq 1$ and $\sum_{i=1}^{\infty} c_{i}=1$ and $\lambda>0$ is the arrival rate of batches. 
b) A single server provides service to all arriving customer, with the service time having general distribution. Let $B(v)$ and $b(v)$ be the distribution and the density function of the service time respectively.

c) we assume interruptions arrive at random while serving the customers and assumed to occur according to a Poisson process with mean rate $\alpha>0$. Let $\beta$ be the server rate of attending interruption. Further we assume that once the interruption arrives the customer whose service is interrupted comes back to the head of the queue. Let $\mu(x) d x$ be the conditional probability of completion of the service during the interval $(x, x+d x]$ given that the elapsed time is $x$, so that

$$
\mu(x)=\frac{b(x)}{1-B(x)},
$$

and therefore,

$$
b(s)=\mu(s) e^{-\int_{0}^{s} \mu(x) d x},
$$

d) As soon as the service is over, the server may take a vacation with probability $\mathrm{p}$ or may continue staying in the system with probability 1-p. After phase one vacation completion the server undergoes phase two and phase three vacation. On completion three heterogeneous phase of vacation the server return back to the system.

e) The server's vacation time follows a general (arbitrary) distribution with distribution function $V_{i}(t)$ and density function $v_{i}(t)$. Let $\gamma_{i}(x) d x$ be the conditional probability of a completion of a vacation during the interval $(x, x+d x]$ given that the elapsed vacation time is $x$, so that

$$
\gamma_{i}(x)=\frac{v_{i}(x)}{1-V_{i}(x)}, \quad i=1,2,3
$$

and therefore,

$$
v_{i}(t)=\gamma_{i}(t) e^{-\int_{0}^{t} \gamma_{i}(x) d x} i=1,2,3 .
$$

f) On returning from vacation the server instantly starts serving the customer at the head of the queue if any.

g) Various stochastic processes involved in the system are assumed to be independent of each other.

\section{DEFINITIONS AND EQUATIONS GOVERNING THE SYSTEM}

We define

$P_{n}(x, t)=$ Probability that at time $t$, the server is active providing essential service and there are $n(n \geq 0)$ customers in the queue excluding the one being served and the elapsed service time for this customer is $x$. Consequently $P_{n}(t)=\int_{0}^{\infty} P_{n}(x, t) d x$ denotes the probability that at time $t$ there are $n$ customers in the queue excluding one customer in the essential service irrespective of the value of $x$

$V_{n}^{(i)}(x, t)=$ Probability that at time $t$, the server is under vacation with elapsed vacation time $x$ and there are $n$ $(n \geq 0)$ customers in the queue. Consequently $V_{n}^{(i)}(t)=$ $\int_{0}^{\infty} V_{n}^{(i)}(x, t) d x$ denotes the probability that at time $t$ there are $n$ customers in the queue and the server is under vacation irrespective of the $x$ for $i=1,2,3$.

$R_{n}(t)=$ Probability that at time $t$, the server is inactive due to the arrival of interruption.

$\mathrm{Q}(\mathrm{t})=$ Probability that at time $t$, there are no customers in the queue or in service and the server is idle but available in the system.

According to the mathematical model mentioned above, the system has the following set of differential-difference equations

$$
\begin{array}{r}
\frac{\partial}{\partial x} P_{0}(x, t)+\frac{\partial}{\partial t} P_{0}(x, t)+[\lambda+\alpha+\mu(x)] P_{0}(x, t)=0 \\
\frac{\partial}{\partial x} P_{n}(x, t)+\frac{\partial}{\partial t} P_{n}(x, t)+[\lambda+\alpha+\mu(x)] P_{n}(x, t)= \\
\lambda \sum_{k=1}^{n} c_{k} P_{n-k}(x, t), \quad n \geq 1
\end{array}
$$

$\frac{\partial}{\partial x} V_{0}^{(1)}(x, t)+\frac{\partial}{\partial t} V_{0}^{(1)}(x, t)+\left[\lambda+\gamma_{1}(x)\right] V_{0}^{(1)}(x, t)=0$

$\frac{\partial}{\partial x} V_{n}^{(1)}(x, t)+\frac{\partial}{\partial t} V_{n}^{(1)}(x, t)+\left[\lambda+\gamma_{1}(x)\right] V_{n}^{(1)}(x, t)=$

$\lambda \sum_{k=1}^{n} c_{k} V_{n-k}^{(1)}(x, t), n \geq 1$

$\frac{\partial}{\partial x} V_{0}^{(2)}(x, t)+\frac{\partial}{\partial t} V_{0}^{(2)}(x, t)+\left[\lambda+\gamma_{2}(x)\right] V_{0}^{(2)}(x, t)=0$

$\frac{\partial}{\partial x} V_{n}^{(2)}(x, t)+\frac{\partial}{\partial t} V_{n}^{(2)}(x, t)+\left[\lambda+\gamma_{2}(x)\right] V_{n}^{(2)}(x, t)=$

$$
\lambda \sum_{k=1}^{n} c_{k} V_{n-k}^{(2)}(x, t), n \geq 1
$$

$\frac{\partial}{\partial x} V_{0}^{(3)}(x, t)+\frac{\partial}{\partial t} V_{0}^{(3)}(x, t)+\left[\lambda+\gamma_{3}(x)\right] V_{0}^{(3)}(x, t)=0$

$\frac{\partial}{\partial x} V_{n}^{(3)}(x, t)+\frac{\partial}{\partial t} V_{n}^{(3)}(x, t)+\left[\lambda+\gamma_{3}(x)\right] V_{n}^{(3)}(x, t)=$

$$
\lambda \sum_{k=1}^{n} c_{k} V_{n-k}^{(3)}(x, t), \quad n \geq 1
$$

$\frac{d}{d t} R_{0}(t)=-(\lambda+\beta) R_{0}(t)$

$$
\begin{aligned}
\frac{d}{d t} R_{n}(t)=-(\lambda+\beta) R_{n}(t) & +\lambda \sum_{k=1}^{n} c_{k} R_{n-k}(t) \\
& +\alpha \int_{0}^{\infty} P_{n-1}(x, t) d x
\end{aligned}
$$

$$
\begin{aligned}
\frac{d}{d t} Q(t)=-\lambda Q(t)+ & \beta R_{0}(t)+\int_{0}^{\infty} \gamma_{3}(x) V_{0}^{(3)}(x, t) d x \\
& +(1-p) \int_{0}^{\infty} \mu(x) P_{0}(x, t) d x
\end{aligned}
$$

Equations are to be solved subject to the following boundary conditions:

$$
\begin{gathered}
P_{n}(0, t)=\lambda c_{n+1} Q(t)+(1-p) \int_{0}^{\infty} \mu(x) P_{n+1}(x, t) d x \\
+\beta R_{n+1}(t)+\int_{0}^{\infty} \gamma_{3}(x) V_{n+1}^{(3)}(x, t) d x, \quad n \geq 0 \\
V_{n}^{(1)}(0, t)=p \int_{0}^{\infty} \mu(x) P_{n}(x, t) d x, n \geq 0 \\
V_{n}^{(2)}(0, t)=\int_{0}^{\infty} \gamma_{1}(x) \bar{V}_{n}^{(1)}(x, t) d x, n \geq 0
\end{gathered}
$$




$$
V_{n}^{(3)}(0, t)=\int_{0}^{\infty} \gamma_{2}(x) \bar{V}_{n}^{(2)}(x, t) d x, \quad n \geq 0
$$

we assume that initially there are no customers in the system and the server is idle. So the initial conditions are

$$
\begin{gathered}
V_{0}^{(i)}(0)=V_{n}^{(i)}(0)=0, Q(0)=1, R_{n}(0)=0 \\
P_{n}(0)=0 \text { for } n \geq 0, \text { and } i=1,2,3 .
\end{gathered}
$$

\section{GENERATING FUNCTIONS OF THE QUEUE LENGTH: THE TIME-DEPENDENT SOLUTION}

In this section we obtain the transient solution for the above set of dfferential-difference equations.

Theorem 4.1 The system of differential difference equations to describe an $M^{[X]} / G / 1$ queue with essential service with service interruption and three phases of vacation are given by equations (1) to (15) with initial conditions (16) and the generating functions of transient solution are given by equation (71) to (75).

Proof :We define the probability generating functions ,

$$
\begin{gathered}
P(x, z, t)=\sum_{n=0}^{\infty} z^{n} P_{n}(x, t), \quad P(z, t)=\sum_{n=0}^{\infty} z^{n} P_{n}(t) \\
R(z, t)=\sum_{n=0}^{\infty} z^{n} R_{n}(t) ; \quad C(z)=\sum_{n=1}^{\infty} c_{n} z^{n} \\
V^{(i)}(x, z, t)=\sum_{n=0}^{\infty} z^{n} V_{n}^{(i)}(x, t), \quad V^{(i)}(z, t)=\sum_{n=0}^{\infty} z^{n} V_{n}^{(i)}(t),
\end{gathered}
$$

which are convergent inside the circle given by $\mathrm{z} \leq 1$ and define the Laplace transform of a function $\mathrm{f}(\mathrm{t})$ as

$$
\bar{f}(s)=\int_{0}^{\infty} e^{-s t} f(t) d t, \Re(s)>0 .
$$

We take the Laplace transform of equations (1) to (15) and using (16), we obtain

$$
\begin{gathered}
\frac{\partial}{\partial x} \bar{P}_{0}(x, s)+(s+\lambda+\alpha+\mu(x)) \bar{P}_{0}(x, s)=0 \\
\frac{\partial}{\partial x} \bar{P}_{n}(x, s)+(s+\lambda+\alpha+\mu(x)) \bar{P}_{n}(x, s)= \\
\lambda \sum_{k=1}^{n} c_{k} \bar{P}_{n-k}(x, s), n \geq 1 \\
\frac{\partial}{\partial x} \bar{V}_{0}^{(1)}(x, s)+\left(s+\lambda+\gamma_{1}(x)\right) \bar{V}_{0}^{(1)}(x, s)=0 \\
\frac{\partial}{\partial x} \bar{V}_{n}^{(1)}(x, s)+\left(s+\lambda+\gamma_{1}(x)\right) \bar{V}_{n}^{(1)}(x, s)= \\
\lambda \sum_{k=1}^{n} c_{k} \bar{V}_{n-k}^{(1)}(x, s), n \geq 1 \\
\frac{\partial}{\partial x} \bar{V}_{0}^{(2)}(x, s)+\left(s+\lambda+\gamma_{2}(x)\right) \bar{V}_{0}^{(2)}(x, s)=0 \\
\frac{\partial}{\partial x} \bar{V}_{n}^{(2)}(x, s)+\left(s+\lambda+\gamma_{2}(x)\right) \bar{V}_{n}^{(2)}(x, s)= \\
\lambda \sum_{k=1}^{n} c_{k} \bar{V}_{n-k}^{(2)}(x, s), n \geq 1
\end{gathered}
$$

$$
\begin{gathered}
\frac{\partial}{\partial x} \bar{V}_{0}^{(3)}(x, s)+\left(s+\lambda+\gamma_{3}(x)\right) \bar{V}_{0}^{(3)}(x, s)=0 \\
\frac{\partial}{\partial x} \bar{V}_{n}^{(3)}(x, s)+\left(s+\lambda+\gamma_{3}(x)\right) \bar{V}_{n}^{(3)}(x, s)= \\
\lambda \sum_{k=1}^{n} c_{k} \bar{V}_{n-k}^{(3)}(x, s), n \geq 1 \\
(s+\lambda+\beta) \bar{R}_{0}(s)=0 \\
(s+\lambda+\beta) \bar{R}_{n}(s)=\lambda \sum_{k=1}^{n} c_{k} \bar{R}_{n-k}(s) \\
+\alpha \int_{0}^{\infty} \bar{P}_{n-1}(x, s) d x, n \geq 1 \\
(s+\lambda) \bar{Q}(s)=1+\beta \bar{R}_{0}(s)+\int_{0}^{\infty} \gamma_{3}(x) \bar{V}_{0}^{(3)}(x, s) d x \\
+(1-p) \int_{0}^{\infty} \mu(x) \bar{P}_{0}(x, s) d x \\
\bar{V}_{n}^{(2)}(0, s)=\int_{0}^{\infty} \bar{V}_{n}^{(1)}(x, s) \gamma_{1}(x) d x, n \geq 0 \\
\bar{V}_{n}^{(1)}(0, s)=\int_{n}^{\infty} \bar{V}_{n}^{(2)}(x, s) \gamma_{2}(x) d x, n \geq 0 . \\
\bar{P}_{n}(0, s)=\lambda c_{n+1} \bar{Q}_{n}(s)+\beta \bar{R}_{n+1}(s)+(1-p) \mu(x) d x, n \geq 0 \\
\bar{P}_{n+1}(x, s) d x+\int_{0}^{\infty} \gamma_{3}(x) \bar{V}_{n+1}^{(3)}(x, s) d x
\end{gathered}
$$

Now multiplying equations (21), (23), (25), (27) by $z^{n}$ and summing over $n$ from 1 to $\infty$, adding to equations (20), (22), (24), (26) and using the generating functions defined in equations (17) and (18) we get

$$
\begin{gathered}
\frac{\partial}{\partial x} \bar{P}_{n}(x, z, s)+[s+\lambda-\lambda C(z)+\alpha+\mu(x)] \bar{P}(x, z, s)=0 \\
\frac{\partial}{\partial x} \bar{V}_{n}^{(1)}(x, z, s)+\left[s+\lambda-\lambda C(z)+\gamma_{1}(x)\right] \bar{V}^{(1)}(x, z, s)=0
\end{gathered}
$$

$\frac{\partial}{\partial x} \bar{V}_{n}^{(2)}(x, z, s)+\left[s+\lambda-\lambda C(z)+\gamma_{2}(x)\right] \bar{V}^{(2)}(x, z, s)=0$

$$
\frac{\partial}{\partial x} \bar{V}_{n}^{(3)}(x, z, s)+\left[s+\lambda-\lambda C(z)+\gamma_{3}(x)\right] \bar{V}^{(3)}(x, z, s)=0
$$

$(s+\lambda-\lambda C(z)+\beta) \bar{R}(z, s)=\alpha z \int_{0}^{\infty} \bar{P}(x, z, s) d x$

For the boundary conditions, we multiply both sides of equation (31) by $z^{n}$ sum over $n$ from 0 to $\infty$, and use the equations (17) and (18) to get

$$
\begin{gathered}
z \bar{P}(0, z, s)=\lambda C(z) \bar{Q}(s)+\beta \bar{R}(z, s)-\beta \bar{R}_{0}(s) \\
+(1-p) \int_{0}^{\infty} \mu(x) \bar{P}(x, z, s) d x-(1-p) \int_{0}^{\infty} \mu(x) \bar{P}_{0}(x, s) d x
\end{gathered}
$$




$$
+\int_{0}^{\infty} \gamma_{3}(x) \bar{V}^{(3)}(x, z, s) d x-\int_{0}^{\infty} \gamma_{3}(x) \bar{V}_{0}(x, s) d x
$$

Using equation (30), equation (40) becomes

$$
\begin{gathered}
z \bar{P}(0, z, s)=[1-s \bar{Q}(s)]+\lambda(C(z)-1) \bar{Q}(s)+\beta \bar{R}(z, s) \\
+\int_{0}^{\infty} \gamma_{3}(x) \bar{V}^{(3)}(x, z, s) d x+(1-p) \int_{0}^{\infty} \mu(x) \bar{P}(x, z, s) d x .
\end{gathered}
$$

Performing similar operation on equations (32) to (34), we get

$$
\begin{gathered}
\bar{V}^{(1)}(0, z, s)=p \int_{0}^{\infty} \mu(x) \bar{P}(x, z, s) d x \\
\bar{V}^{(2)}(0, z, s)=\int_{0}^{\infty} \gamma_{1}(x) \bar{V}^{(1)}(x, z, s) d x \\
\bar{V}^{(3)}(0, z, s)=\int_{0}^{\infty} \gamma_{2}(x) \bar{V}^{(2)}(x, z, s) d x
\end{gathered}
$$

Integrating equation (35) between 0 to $x$, we get

$$
\bar{P}(x, z, s)=\bar{P}(0, z, s) e^{-[s+\lambda-\lambda C(z))+\alpha] x-\int_{0}^{x} \mu(t) d t}
$$

where $\bar{P}(0, z, s)$ is given by equation (41). Again integrating equation (45) by parts with respect to $x$ yields,

$$
\bar{P}(z, s)=\bar{P}(0, z, s)\left[\frac{1-\bar{B}(s+\lambda-\lambda C(z)+\alpha)}{s+\lambda-\lambda C(z)+\alpha}\right]
$$

where

$$
\bar{B}(s+\lambda-\lambda C(z)+\alpha)=\int_{0}^{\infty} e^{-[s+\lambda-\lambda C(z)+\alpha] x} d B(x)
$$

is the Laplace-Stieltjes transform of the essential service time $B(x)$. Now multiplying both sides of equation (45) by $\mu(x)$ and integrating over $x$ we obtain

$$
\int_{0}^{\infty} \bar{P}(x, z, s) \mu(x) d x=\bar{P}(0, z, s) \bar{B}[s+\lambda-\lambda C(z)+\alpha]
$$

Similarly, on integrating equations (36) to (38) from 0 to $x$, we get

$$
\begin{aligned}
& \bar{V}^{(1)}(x, z, s)=\bar{V}^{(1)}(0, z, s) e^{-[s+\lambda-\lambda C(z)] x-\int_{0}^{x} \gamma_{1}(t) d t} \\
& \bar{V}^{(2)}(x, z, s)=\bar{V}^{(2)}(0, z, s) e^{-[s+\lambda-\lambda C(z)] x-\int_{0}^{x} \gamma_{2}(t) d t} \\
& \bar{V}^{(3)}(x, z, s)=\bar{V}^{(3)}(0, z, s) e^{-[s+\lambda-\lambda C(z)] x-\int_{0}^{x} \gamma_{3}(t) d t}
\end{aligned}
$$

where $\bar{V}^{(1)}(0, z, s), \quad \bar{V}^{(2)}(0, z, s)$, and $\bar{V}^{(3)}(0, z, s)$ are given by equations (42) to (44). Again integrating equations (49) to (51) by parts with respect to $x$ yields,

$$
\begin{aligned}
& \bar{V}^{(1)}(z, s)=\bar{V}^{(1)}(0, z, s)\left[\frac{1-\bar{V}_{1}(s+\lambda-\lambda C(z))}{s+\lambda-\lambda C(z)}\right] \\
& \bar{V}^{(2)}(z, s)=\bar{V}^{(2)}(0, z, s)\left[\frac{1-\bar{V}_{2}(s+\lambda-\lambda C(z))}{s+\lambda-\lambda C(z)}\right] \\
& \bar{V}^{(3)}(z, s)=\bar{V}^{(3)}(0, z, s)\left[\frac{1-\bar{V}_{3}(s+\lambda-\lambda C(z))}{s+\lambda-\lambda C(z)}\right]
\end{aligned}
$$

where

$$
\bar{V}_{1}(s+\lambda-\lambda C(z))=\int_{0}^{\infty} e^{-[s+\lambda-\lambda C(z)] x} d V_{1}(x)
$$

$$
\begin{aligned}
& \bar{V}_{2}(s+\lambda-\lambda C(z))=\int_{0}^{\infty} e^{-[s+\lambda-\lambda C(z)] x} d V_{2}(x) \\
& \bar{V}_{3}(s+\lambda-\lambda C(z))=\int_{0}^{\infty} e^{-[s+\lambda-\lambda C(z)] x} d V_{3}(x)
\end{aligned}
$$

is the Laplace-Stieltjes transform of the first phase, second phase and third phase of vacation time $V_{1}(x), V_{2}(x)$ and $V_{3}(x)$ respectively. Now multiplying both sides of equations (49), (50), (51) by $\gamma_{1}(x), \gamma_{2}(x)$ and $\gamma_{3}(x)$ and integrating over $x$ we obtain

$\int_{0}^{\infty} \bar{V}^{(1)}(x, z, s) \gamma_{1}(x) d x=\bar{V}^{(1)}(0, z, s) \bar{V}_{1}[s+\lambda-\lambda C(z)]$

$\int_{0}^{\infty} \bar{V}^{(2)}(x, z, s) \gamma_{2}(x) d x=\bar{V}^{(2)}(0, z, s) \bar{V}_{2}[s+\lambda-\lambda C(z)]$

$\int_{0}^{\infty} \bar{V}^{(3)}(x, z, s) \gamma_{3}(x) d x=\bar{V}^{(3)}(0, z, s) \bar{V}_{3}[s+\lambda-\lambda C(z)]$

Using equation (48) in equation (42), we get

$$
\bar{V}^{(1)}(0, z, s)=p \bar{B}(s+\lambda-\lambda C(z)+\alpha) \bar{P}(0, z, s)
$$

Now using equations (58) and (61) in (43), we get

$$
\begin{gathered}
\bar{V}^{(2)}(0, z, s)=p \bar{V}_{1}(s+\lambda-\lambda C(z)) \\
\bar{B}(s+\lambda-\lambda C(z)+\alpha) \bar{P}(0, z, s)
\end{gathered}
$$

By using equations (59) and (62) in (44), we get

$$
\begin{array}{r}
\bar{V}^{(3)}(0, z, s)=p \bar{V}_{1}(s+\lambda-\lambda C(z)) \bar{V}_{2}(s+\lambda-\lambda C(z)) \\
\bar{B}(s+\lambda-\lambda C(z)+\alpha) \bar{P}(0, z, s)
\end{array}
$$

Using equation (48), (60) and (63) in (41), we get

$$
\begin{gathered}
{\left[z-p \bar{V}_{1} \bar{V}_{2} \bar{V}_{3} \bar{B}-(1-p) \bar{B}\right] \bar{P}(0, z, s)=[1-s \bar{Q}(s)]} \\
+\lambda(C(z)-1) \bar{Q}(s)+\beta \bar{R}(z, s)
\end{gathered}
$$

From (39) and (45), we get

$$
\begin{aligned}
\bar{R}(z, s)=\frac{\alpha z}{s+\lambda-\lambda C(z)+\beta} \bar{P}(0, z, s) \\
\\
{\left[\frac{1-\bar{B}(s+\lambda-\lambda C(z)+\alpha}{s+\lambda-\lambda C(z)+\alpha}\right] }
\end{aligned}
$$

where $\bar{B}=\bar{B}(\lambda-\lambda C(z)+\alpha), \bar{V}_{1}=\bar{V}_{1}(\lambda-\lambda C(z))$, $\bar{V}_{2}=\bar{V}_{2}(\lambda-\lambda C(z))$ and $\bar{V}_{3}=\bar{V}_{3}(\lambda-\lambda C(z))$.

Now using equation (65) in (64), we have

$$
\bar{P}(0, z, s)=\frac{f_{1}(z) f_{2}(z)[(1-s \bar{Q}(s))+\lambda(C(z)-1) \bar{Q}(s)]}{d r}
$$

Similarly using equation (66), in equations (61), (62) and (63), we get

$\bar{V}^{(1)}(0, z, s)=\frac{p \bar{B} f_{1}(z) f_{2}(z)[(1-s \bar{Q}(s))+\lambda(C(z)-1) \bar{Q}(s)]}{d r}$

$$
\begin{aligned}
& \bar{V}^{(2)}(0, z, s)=\frac{p \bar{V}_{1} \bar{B} f_{1}(z) f_{2}(z)}{d r} \\
& {[(1-s \bar{Q}(s))+\lambda(C(z)-1) \bar{Q}(s)] } \\
& \bar{V}^{(3)}(0, z, s)=\frac{p \bar{V}_{1} \bar{V}_{2} \bar{B} f_{1}(z) f_{2}(z)}{d r} \\
& {[(1-s \bar{Q}(s))+\lambda(C(z)-1) \bar{Q}(s)] }
\end{aligned}
$$

where

$d r=f_{1}(z) f_{2}(z)\left[z-p \bar{V}_{1} \bar{V}_{2} \bar{V}_{3} \bar{B}-(1-p) \bar{B}\right]-\alpha z \beta(1-\bar{B})$, 
$f_{1}(z)=s+\lambda-\lambda C(z)+\beta$, and $f_{2}(z)=s+\lambda-\lambda C(z)+\alpha$.

Using equations (66) to (69) in equations (46), (52), (53), (54) and (65), we get

$$
\begin{gathered}
\bar{P}(z, s)=\frac{\left.f_{1}(z)\right)[1-s \bar{Q}(s)+\lambda(C(z)-1) \bar{Q}(s)][1-\bar{B}]}{d r} \\
\bar{V}^{(1)}(z, s)=\frac{[1-s \bar{Q}(s)+\lambda(C(z)-1) \bar{Q}(s)]}{d r} \\
\bar{V}^{(2)}(z, s)=\frac{[1-s \bar{Q}(s)+\lambda(C(z)-1) \bar{Q}(s)]}{d r} \\
p \bar{B} \bar{V}_{1} f_{1}(z) f_{2}(z)\left[\frac{1-V_{2}(s+\lambda-\lambda C(z))}{s+\lambda-\lambda C(z)}\right] \\
\bar{V}^{(3)}(z, s)=\frac{[(1-s \bar{Q}(s))+\lambda(C(z)-1) \bar{Q}(s)]}{d r} \\
p \bar{B} \bar{V}_{1} \bar{V}_{2} f_{1}(z) f_{2}(z)\left[\frac{1-V_{1}(s+\lambda-\lambda C(z))}{s+\lambda+\lambda-\lambda C(z))}\right] \\
\bar{R}(z, s)=\frac{\alpha z(1-\bar{B})[1-s \bar{Q}(s)+\lambda(C(z)-1) \bar{Q}(s)]}{d r}
\end{gathered}
$$

where $\mathrm{dr}$ is given by equation (70). Thus $\bar{P}(z, s), \quad \bar{V}^{(1)}(z, s), \quad \bar{V}^{(2)}(z, s), \quad \bar{V}^{(3)}(z, s)$ and $\bar{R}(z, s)$ are completely determined from equations (71) to (75) which completes the proof of the theorem.

\section{THE STEADY STATE RESULTS}

In this section, we shall derive the steady state probability distribution for our queueing model. These probabilities are obtained by suppressing the argument $t$ wherever it appears in the timedependent analysis. This can be obtained by applying the wellknown Tauberian property,

$$
\lim _{s \rightarrow 0} s \bar{f}(s)=\lim _{t \rightarrow \infty} f(t)
$$

In order to determine $\bar{P}(z, s), \bar{V}^{(1)}(z, s), \bar{V}^{(2)}(z, s), \bar{V}^{(3)}(z, s)$ and $\bar{R}(z, s)$ completely, we have yet to determine the unknown $\mathrm{Q}$ which appears in the numerators of the right hand sides of equations (71) to (75). For that purpose, we shall use the normalizing condition

$$
P(1)+V^{(1)}(1)+V^{(2)}(1)+V^{(3)}(1)+R(1)+Q=1
$$

Theorem 5.1 The steady state probabilities for an $M^{[X]} / G / 1$ queue with service interruption and three phases vacation are given by

$$
\begin{aligned}
P(1) & =\frac{\lambda E(I) \beta[1-\bar{B}(\alpha)] Q}{D R} \\
V^{(1)}(1) & =\frac{\lambda p \alpha \beta E(I) \bar{B}(\alpha) E\left(V_{1}\right) Q}{D R} \\
V^{(2)}(1) & =\frac{\lambda p \alpha \beta E(I) \bar{B}(\alpha) E\left(V_{2}\right) Q}{D R} \\
V^{(3)}(1) & =\frac{\lambda p \alpha \beta E(I) \bar{B}(\alpha) E\left(V_{3}\right) Q}{D R} \\
R(1) & =\frac{\lambda \alpha E(I)[1-\bar{B}(\alpha)] Q}{D R}
\end{aligned}
$$

where

$D R=\alpha \beta \bar{B}(\alpha)[1-\lambda E(I) p E(V)]-\lambda E(I)(\alpha+\beta)[1-\bar{B}(\alpha)]$,

$\mathrm{E}(\mathrm{V})=E\left(V_{1}\right)+E\left(V_{2}\right)+E\left(V_{3}\right), P(1), V^{(1)}(1), V^{(2)}(1), V^{(3)}(1)$, $R(1)$ and $\mathrm{Q}$ are the steady state probabilities that the server is providing essential service, first phase of vacation, second phase of vacation, third phase of vacation and server under idle respectively without regard to the number of customers in the queue.

Proof: Multiplying both sides of equations (71) to (75) by $\mathrm{s}$, taking limit as $s \rightarrow 0$, applying Tauberian property and simplifying, we obtain

$$
\begin{gathered}
P(z)=\frac{f_{3}(z)(1-\bar{B}) \lambda(C(z)-1) Q}{d r} \\
V^{(1)}(z)=\frac{p f_{3}(z) f_{4}(z) \bar{B}\left[\bar{V}_{1}-1\right] Q}{d r} \\
V^{(2)}(z)=\frac{p f_{3}(z) f_{4}(z) \bar{V}_{1} \bar{B}\left[\bar{V}_{2}-1\right] Q}{d r} \\
V^{(3)}(z)=\frac{p f_{3}(z) f_{4}(z) \bar{V}_{1} \bar{V}_{2} \bar{B}\left[\bar{V}_{3}-1\right] Q}{d r} \\
R(z)=\frac{\alpha z(1-\bar{B}) \lambda(C(z)-1) Q}{d r}
\end{gathered}
$$

where $\mathrm{dr}$ is given by equation (70), $f_{3}(z)=\lambda-\lambda C(z)+$ $\beta, f_{4}(z)=\lambda-\lambda C(z)+\alpha$,

Let $W_{q}(z)$ denote the probability generating function of the queue size irrespective of the state of the system. Then adding equations (84) to (88)we obtain

$$
\begin{aligned}
W_{q}(z)=P^{(1)}(z) & +P^{(2)}(z)+P^{(3)}(z)+V^{(1)}(z)+V^{(2)}(z) \\
W_{q}(z) & =\frac{f_{3}(z)(1-\bar{B}) \lambda(C(z)-1) Q}{d r} \\
+ & \frac{p f_{3}(z) f_{4}(z) \bar{B}\left[\bar{V}_{1}-1\right] Q}{d r} \\
+ & \frac{p f_{3}(z) f_{4}(z) \bar{V}_{1} \bar{B}\left[\bar{V}_{2}-1\right] Q}{d r} \\
& +\frac{p f_{3}(z) f_{4}(z) \bar{V}_{1} \bar{V}_{2} \bar{B}\left[\bar{V}_{3}-1\right] Q}{d r} \\
& +\frac{\alpha z(1-\bar{B}) \lambda(C(z)-1) Q}{d r}
\end{aligned}
$$

We see that for $z=1, W_{q}(1)$ is indeterminate of the form $0 / 0$. Therefore, we apply L'Hopital's rule and on simplifying we obtain the result (89), where $\mathrm{C}(1)=1, C^{\prime}(1)=E(I)$ is mean batch size of the arriving customers, $-\bar{B}^{\prime}(0)=E(B), \quad-\bar{V}_{i}^{\prime}(0)=$ $E\left(V_{i}\right), i=1,2,3$.

$W_{q}(1)=\frac{\lambda E(I)(\alpha+\beta)[1-\bar{B}(\alpha)]+\lambda E(I) p \alpha \beta \bar{B}(\alpha) E(V) Q}{D R}$

and DR is given by equation (83). Therefore adding $\mathrm{Q}$ to equation (90), equating to 1 and simplifying, we get

$$
Q=1-\rho
$$

and hence the utilization factor $\rho$ of the system is given by

$$
\rho=\lambda p E(I) E(V)-\frac{\lambda E(I)}{\bar{B}(\alpha)}\left(\frac{1}{\beta}+\frac{1}{\alpha}\right)[1-\bar{B}(\alpha)]
$$

where $\rho<1$ is the stability condition under which the steady state exists. Equation (91) gives the probability that the server is idle. Substituting Q from (91) into (89), we have completely and explicitly determined $W_{q}(z)$, the probability generating function of the queue size. 


\section{THE AVERAGE QUEUE SIZE}

Let $L_{q}$ denote the average number of customers in the queue under the steady state. Then

$$
L_{q}=\frac{d}{d z} W_{q}(z) \text { at } z=1
$$

Since this formula gives $0 / 0$ form, then we write $W_{q}(z)$ given in (89) as $W_{q}(z)=\frac{N(z)}{D(z)}$ where $N(z)$ and $D(z)$ are numerator and denominator of the right hand side of (89) respectively. Then we use

$$
L_{q}=\lim _{z \rightarrow 1} \frac{d}{d z} W_{q}(z)=\lim _{z \rightarrow 1}\left[\frac{D^{\prime}(1) N^{\prime \prime}(1)-N^{\prime}(1) D^{\prime \prime}(1)}{2\left(D^{\prime}(1)\right)^{2}}\right]_{Q}
$$

where primes and double primes in (93) denote first and second derivative at $z=1$, respectively. Carrying out the derivative at $z$ $=1$ we have

$$
\begin{gathered}
N^{\prime}(1)=\lambda E(I)(\alpha+\beta)[1-\bar{B}(\alpha)]+\lambda p \alpha \beta E(I) \bar{B}(\alpha) E(V) \\
N^{\prime \prime}(1)=\lambda[1-\bar{B}(\alpha)][E(I(I-1))(\alpha+\beta)+2 E(I)(-\lambda E(I)+\alpha)] \\
+2 \lambda^{2}(E(I))^{2}(\alpha+\beta) \bar{B}^{\prime}(\alpha)-2 \lambda^{2}(E(I))^{2} p E(V) \\
{\left[(\alpha+\beta) \bar{B}(\alpha)+\alpha \beta \bar{B}^{\prime}(\alpha)\right]+\lambda p \alpha \beta E(I(I-1)) \bar{B}(\alpha) E(V)} \\
+\lambda^{2}(E(I))^{2} p \alpha \beta \bar{B}(\alpha)\left[E\left(V_{1}^{2}\right)+2 E\left(V_{1}\right)\left(E\left(V_{2}\right)+E\left(V_{3}\right)\right)\right. \\
\left.\quad+E\left(V_{2}^{2}\right)+2 E\left(V_{2}\right) E\left(V_{3}\right)+E\left(V_{3}^{2}\right)\right] \\
D^{\prime}(1)=\alpha \beta \bar{B}(\alpha)[1-\lambda E(I) p E(V)-\lambda E(I)(\alpha+\beta)[1-\bar{B}(\alpha)] \\
D^{\prime \prime}(1)=\left[-\lambda(\alpha+\beta) E(I(I-1))+2 \lambda^{2}(E(I))^{2}\right][1-\bar{B}(\alpha)] \\
-2 \lambda E(I)(\alpha+\beta)\left[1-\lambda E(I) p \bar{B}(\alpha) E(V)+\lambda E(I) \bar{B}^{\prime}(\alpha)\right] \\
-\lambda p \alpha \beta E(I(I-1)) \bar{B}(\alpha) E(V)-2 \lambda \alpha \beta E(I) \bar{B}^{\prime}(\alpha) \\
\quad-\lambda^{2} \alpha \beta p(E(I))^{2} \bar{B}(\alpha)\left[E\left(V_{1}^{2}\right)+E\left(V_{2}^{2}\right)+E\left(V_{3}^{2}\right)\right] \\
-2 \lambda^{2} \alpha \beta p(E(I))^{2} \bar{B}(\alpha)\left[E\left(V_{1}\right) E\left(V_{2}\right)+E\left(V_{1}\right) E\left(V_{3}\right)\right. \\
\left.+E\left(V_{2}\right) E\left(V_{3}\right)\right]+2 \lambda^{2} \alpha \beta p(E(I))^{2} \bar{B}^{\prime}(\alpha) E(V)
\end{gathered}
$$

where $E\left(V^{2}\right)$ are the second moment of the vacation time. $E(I(I-1))$ is the second factorial moment of the batch size of arriving customers. Then if we substitute the values $N^{\prime}(1), N^{\prime \prime}(1), D^{\prime}(1), D^{\prime \prime}(1)$ from equations (94) to (97) into equations (93) we obtain $L_{q}$ in the closed form. Further, we find the mean system size L using Little's formula. Thus we have

$$
L=L_{q}+\rho
$$

where $L_{q}$ has been found by equation (93) and $\rho$ is obtained from equation (92).

\section{THE AVERAGE WAITING TIME}

Let $W_{q}$ and $\mathrm{W}$ denote the mean waiting time in the queue and in the system respectively. Then using Little's formula, we obtain,

$$
\begin{gathered}
W_{q}=\frac{L_{q}}{\lambda} \\
W=\frac{L}{\lambda}
\end{gathered}
$$

Where $L_{q}$ and $\mathrm{L}$ have been found in equations (93) and (98).

\section{PARTICULAR CASE}

If $E\left(V_{2}\right)=E\left(V_{3}\right)=0, \mathrm{E}(\mathrm{I})=1, \mathrm{E}(\mathrm{I}(\mathrm{I}-1))=0$, our model reduces to a single server $\mathrm{M} / \mathrm{G} / 1$ queue with service interruption and Bernoulli schedule server vacation. In this case, the probability generating function of the number of customers in the queue $W_{q}(z)$, the idle probability $\mathrm{Q}$ and the average queue size $L_{q}$ can be simplified to the following expressions and the equations coincides with equation of Balamani [3].

$$
\begin{gathered}
Q=1-\lambda p E\left(V_{1}\right)-\frac{\lambda}{\bar{B}(\alpha)}\left(\frac{1}{\beta}+\frac{1}{\alpha}\right)(1-\bar{B}(\alpha)) \\
W_{q}(z)=\frac{\lambda(z-1)(1-\bar{B})\left[f_{1}(z)+\alpha z\right]+p f_{1}(z) f_{2}(z) \bar{B}[\bar{V}-1]}{f_{1}(z) f_{2}(z)[z-p \bar{B} \bar{V}-(1-p) \bar{B}]-\alpha z \beta(1-\bar{B})} Q \\
L_{q}=\lim _{z \rightarrow 1}\left[\frac{D^{\prime}(1) N^{\prime \prime}(1)-N^{\prime}(1) D^{\prime \prime}(1)}{2\left(D^{\prime}(1)\right)^{2}}\right] Q \\
N^{\prime}(1)=\lambda(\alpha+\beta)(1-\bar{B}(\alpha))+\lambda p \alpha \beta \bar{B}(\alpha) E\left(V_{1}\right) \\
N^{\prime \prime}(1)=2 \lambda(-\lambda+\alpha)(1-\bar{B}(\alpha))+2 \lambda^{2}(\alpha+\beta) \bar{B}^{\prime}(\alpha) \\
\quad-2 \lambda^{2} p E\left(V_{1}\right)[(\alpha+\beta) \bar{B}(\alpha)+\alpha \beta \bar{B}(\alpha)] \\
\quad+\lambda^{2} p \alpha \beta \bar{B}(\alpha) E\left(V_{1}^{2}\right) \\
D^{\prime}(1)= \\
\begin{aligned}
D^{\prime \prime}(1)= & 2 \lambda^{2}\left(1-\bar{B}(\alpha)\left(1-\lambda p E\left(V_{1}\right)\right)-\lambda(\alpha+\beta)\right)+2 \lambda^{2} \alpha \beta p \bar{B}^{\prime}(\alpha) E\left(V_{1}\right) \\
& -2 \lambda(\alpha+\beta)\left[1-\lambda p \bar{B}(\alpha) E\left(V_{1}\right)+\lambda \bar{B}^{\prime}(\alpha)\right] \\
& -\lambda^{2} \alpha \beta p \bar{B}^{\prime}(\alpha) E\left(V_{1}^{2}\right)-2 \lambda \alpha \beta \bar{B}^{\prime}(\alpha)
\end{aligned}
\end{gathered}
$$

\section{CONCLUSION}

In this paper we have studied a batch arrival, essential service with interruption and three phases of vacation. This paper clearly analyzes the transient solution, steady state results of our queueing system. As a future work busy period analysis and reliability analysis will be discussed.

Acknowledgements The authors are thankful to the referees for their valuable comments and suggestions to improve the quality of the paper.

\section{REFERENCES}

[1] Avi Itzhak, B. and Naor, P. 1963. Some queueing problems with the service station subject to breakdowns, Operations Research , 11, 303 - 319.

[2] Baskar, S. Rajalakshmi Rajagopal and Palaniammal, S. 2011. A Single server M/G/1 queue with service interruption under bernoulli schedule, International Mathematical Forum, 6, 1697 - 1712

[3] Balamani, N. 2012. A single server M/G/1 queue with service interruption and bernoulli schedule server vacation having general vacation time distribution, International Journal of Mathematical Archive, 3(2), 347-353.

[4] Borthakur, A. and Choudhury, G. 1997. On a batch arrival Poisson queue with generalised vacation, Sankhya Ser. B, 59, 369-383.

[5] Doshi, B. 1986. Queueing systems with vacations-a survey, Queueing Systems, 1, 29-66.

[6] Kulkarni, V. G. and Choi, B. D. 1990. Retrial queues with server subject to breakdowns and repairs, Queueing Systems, 7, 191-209. 
[7] Madan, K. C., Abu-Dayyeh, W. Gharaibeh, M. 2003. Steady state analysis of two $M^{[x]} / M^{[a, b]} / 1$ queue models with random breakdowns, International Journal of Information and Management Sciences, 14, 37-51.

[8] Maraghi, F. A., Madan, K. C. and Darby-Dowman, K. 2009. Batch arrival queueing system with random breakdowns and Bernoulli schedule server vacations having general vacation time distribution, International Journal of Information and Management Sciences, 20, 55-70.

[9] Takagi, H. 1991. Queueing Analysis: A Foundation of performance evaluation, Vol. 1: Vacation and priority systems, Part 1, Elsevier Science Publishers, Amsterdam,

[10] Takine, T. and Sengupta, B. 1997. A single server queue with service interruptions, Queueing Systems, 26(3), 285300 .

[11] Thangaraj, V. and Vanitha, S. 2010. M/G/1 queue with twostage heterogeneous service compulsory server vacation and random breakdowns, Int. J. Contemp. Math. Sciences, 5, 307-322.

[12] Thiruvengadam, K. 1963. Queueing with breakdowns, Operations Research 11, 62-71.

[13] Tian, N. and and Zhang, G. 2006. Vacation queueing models - Theory and Applications, Springer-Verlag, New York.

[14] Tian, N., Li, Q. and Cao, J. 1999. Conditional stochastic decomposition in $\mathrm{M} / \mathrm{M} / \mathrm{c}$ queue with server vacations, Stochastic Models, 15, 367-377.

[15] Wang, J., Cao, J. and Li, Q. 2001. Reliability analysis of the retrial queue with server breakdowns and repairs, Queueing Systems, 38, 363-380.

[16] White, H. and Christie, L. 1985. Queueing with preemptive priorities or with breakdowns, Operations Research, 6, 7995. 